\title{
Workshop on Web Information Seeking and Interaction
}

\author{
Kerry Rodden \\ Google \\ Mountain View, CA 94043, USA \\ kerryr@google.com
}

\author{
Ian Ruthven \\ University of Strathclyde \\ Glasgow, G1 1XH, UK \\ ir@cis.strath.ac.uk
}

\author{
Ryen W. White \\ Microsoft Research \\ Redmond, WA 98052, USA \\ ryenw@microsoft.com
}

\section{Introduction}

The World Wide Web has provided access to a diverse range of information sources and systems. People engaging with this rich network of information may need to interact with different technologies, interfaces, and information providers in the course of a single search task. These systems may offer different interaction affordances and require users to adapt their informationseeking strategies. Not only is this challenging for users, but it also presents challenges for the designers of interactive systems, who need to make their own system useful and usable to broad user groups. The popularity of Web browsing and Web search engines has given rise to distinct forms of information-seeking behaviour, and new interaction styles, but we do not yet fully understand these or their implications for the development of new systems.

Web Information Seeking and Interaction (WISI) (i.e., the interaction of users with Web-based content and applications during information-seeking activities) is a topic that unites many strands of academic and commercial research, from studies of information-seeking behaviour to the design and construction of large-scale interactive systems. Designing components to support this interaction (and evaluating these components) is particularly challenging given the scale of the Web, the diversity of the user population, the diversity in tasks being undertaken, and the dynamic nature of the information. This workshop acted as a focal point for researchers and practitioners whose work is related to Web information seeking and interaction, to enable them to share experiences and collaborate.

Papers submitted to the workshop were reviewed by an international programme committee and 16 short papers and position papers were accepted for the workshop proceedings. These papers reflect the diversity of interest and contributions to the area of Web information seeking and interaction, describing research on evaluation, interaction modelling, information behaviour, personalisation, document surrogates, and novel interface development, amongst other topics. The proceedings are available at http://research.microsoft.com/ ryenw/proceedings/WISI2007.pdf.

\section{Panel}

The workshop began with a panel on "Challenges and Opportunities in Supporting Web Search Interaction". We were fortunate to have three interesting speakers: Peter Anick (Yahoo!), Diane Kelly (University of North Carolina), and Maria Stone (Google). What follows is our personal interpretation of the panel session and discussion. 
Maria Stone has led the user experience research team at Google for six years. Maria covered usability and evaluation issues from a Web search engine perspective. Web search engine developers and evaluators face similar problems to other practitioners with a generally unknown, and diverse user population, and an extremely diverse set of data. Data quality itself is the biggest issue for search engine developers. In her panel address Maria started off by reminding us that many Google users are actually infrequent users who, although they may have complex information needs, interact in very simple ways with the tools available. Major challenges are, therefore, understanding what users want when searching and also how to best to support their searching through new search features. A separate, related, challenge is how to provide these features in a way that does not reduce the efficiency of current Web search tools.

Peter Anick presented three main challenges to developing new methods of interaction: discoverability, content, and learnability. He presented a fascinating characterisation of typical search engine users, characterising them as generally:

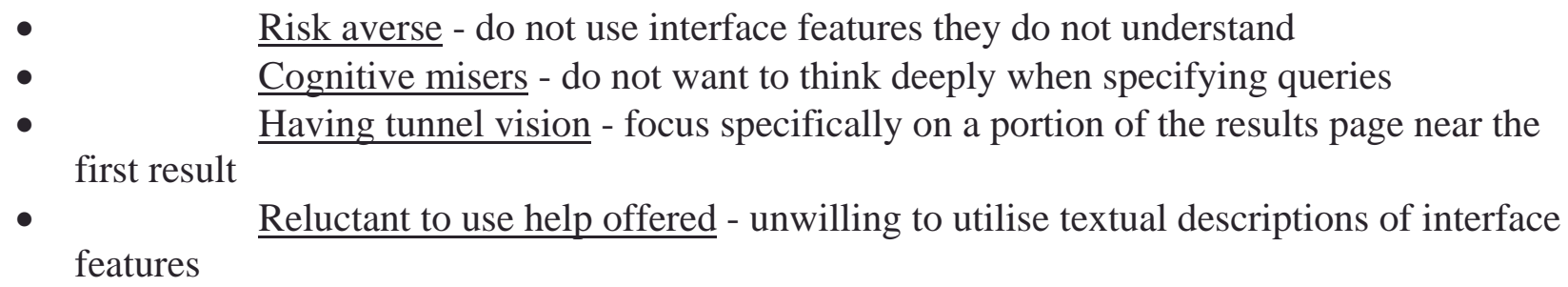

This should not be seen as a negative characterisation, but rather a set of generalisations about typical interactions with a search engine. Such behaviour raises interesting challenges, including how to empower users to have more control and input into their interaction, how to cope with different types of searches (such as exploratory search), and what tools are appropriate for investigation of behaviour and evaluation. Peter also gave an interesting demo on the some of the to-be-released interaction features from Yahoo's "search assist" tool which led to important discussion on how to encourage searchers to provide more information to the search system.

Diane Kelly then turned the discussion to the fundamentals of Web information seeking and interaction by presenting a series of questions about the direction and nature of the field. In the WISI area, for example, what kind of activities are we (and should we) be engaged in? Are we, for example, really trying to learn about users and their needs or just treating users as black boxes and examining only their output? Are any lessons we learn from user studies generalisable into solid principles that can be applied in different situations? A core question posed is whether the field is only an applied science - or whether it is even a science at all. Certainly the area of search systems itself has many applications, but what about the methodologies and models we develop - are these good science? These questions have many implications for the community, how it develops, and how it evaluates its own progress.

\section{Breakout sessions}

The panel was followed by breakout sessions where participants were asked to form small groups and present solutions to problems set by the panelists and organisers. Each group was asked to discuss the problem and report.

Two main issues arose from the discussions: (i) tasks and (ii) improving the search experience. 
Tasks: Many search engines perform well for some types of tasks. For example, Google is generally excellent for fact-finding searches. However other types of task require increased support. Knowing more about which tasks require such support and what type of support would be useful was a central issue across the discussions.

Improving the search experience: If we are to improve search engine effectiveness across a range of tasks, how might this be achieved in practice? Are better systems or better interfaces more fruitful areas of investigation?

Tackling the overall search experience was an important issue. One group, whose conclusions were presented by Mark Smucker of UMass, identified a spectrum of possible solutions, from manuallyled approaches (based on improved information literacy and teaching) through to automatic, systembased, approaches (based on more intelligent systems). We coarsely present this useful spectrum below.

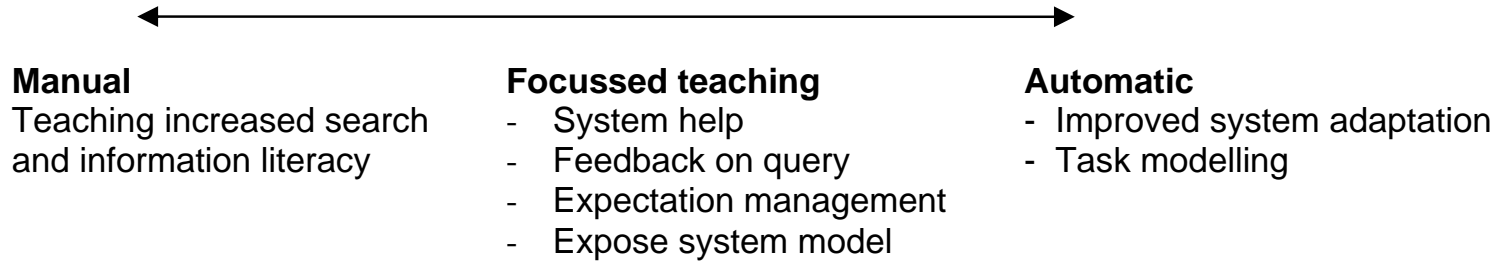

One implication of this spectrum is that not all advances in search engine effectiveness need be those arising from technical developments. That is, education and awareness of existing tools can be crucial to successful searching.

\section{Presented papers}

The first afternoon session consisted of short paper presentations. We selected 4 papers from those appearing in the proceedings that reflected common themes within the papers submitted for the workshop.

Shady Elbassuoni presented his paper Adaptive Personalization of Web Search, co-authored with Julia Luxenburger and Gerhard Weikum, which proposed a system architecture for adapting search results to a user's search tasks. This client-side model captures user interactions, specifically HTTP traffic, so that the personalisation system can capture user queries, result clicks and page visits. Information on previous searches is used to predict what type of search a user is carrying out and reorder search results to adapt to that type of search. A repeat query, for example, is seen an attempt to re-find previously seen information and are prioritised in the results list. A comparison with a nonadaptive search engine shows promising results.

Kerry Rodden presented Exploring How Mouse Movements Relate to Eye Movements on Web Search Results Pages, co-authored with Xin Fu. The study presented in this paper attempted to correlate mouse movements (as opposed to simple mouse clicks) with eye-tracking data. In this exploratory study eye movements and mouse movements did show a reasonably good correlation. The study also showed interesting behaviours with the mouse. For example a small number of participants used the mouse pointer as a reading aid, moving the mouse across the text they were reading. Other 
participants left the mouse pointer at an interesting result in order to 'mark' the result whilst they scanned other results. Work such as this open up new ways of measuring interactive search behaviour and giving new insights into online searching.

Elaine Toms presented Evaluating Engagement in Interactive Search co-authored with Heather O'Brien in which they propose user engagement as a new metric for evaluating interactive search systems. Engagement here represents a higher-order construct that expresses the level of positive involvement in a task or process. Engagement is a compound construct and Toms and O'Brien discuss the methodological issues involved in estimating such a complex, but useful, metric both in terms of measuring the outcome - how engaged were users in a search - and the process of searching - how does engagement change and manifest itself during a period of interaction?

The final paper titled "Clickthrough-based Measures of Search Engine Performance" which was coauthored by Craig Macdonald, and Iadh Ounis was presented by Erik Graf. Within this paper they examined an important issue in interactive information seeking and search, namely how to model interactive search behaviour. They present a framework for modelling interactive search behaviour based on a taxonomy that arranges loggable interaction features such as the query, user ID, and query submission timestamp into user related, request related, or result related groups. This framework, instantiated within an intranet retrieval system, was used to test various hypotheses about user interaction. One of which was for example whether clickthrough behaviour can be used to infer the success of search sessions? A particular example presented to outline the potential of their approach lay in the successful identification of a period of system failure via analysis of interaction patterns.

\section{Discussion}

Our workshop ended with a plenary discussion on the themes arising from the discussions throughout the event, the background papers, and breakout sessions. Here we summarise some of these themes and the discussions arising.

- How to get more from users about what they want and how do we obtain this information in a way that that involves the least intrusion? Web search engines have provided simple models of interaction and users have learnt a particular model of interaction. Going beyond this, by asking users to provide more information themselves, may be difficult unless we can, at the same time, make clear the benefits of providing more information.

- Diane Kelly, in her panel address, raised the question of what type of science(s) we are engaged in. One point raised in response was that our field has an increasingly heavy industrial component and both sides of the academic/industrial fence need guidance on what the other side wants. From the audience, the academics were clear that they wanted data whereas practioners wanted general, coherent principles of interaction.

- What concepts are (most) useful for investigation? A recurring concept was that of task, reflecting the increased interest in this concept within the community. This led into a discussion of the granularity of investigation appropriate for learning about searchers and search behaviour of the individual characteristics that are important in search interaction such as age, information literacy, and information poverty. The issue of information literacy led back to a discussion on the education of users - most Web users have little training in online searching - and the possible dangers of users passively accepting search results.

- The issue of success and user satisfaction was another recurring theme throughout the day. Participants from a background in more traditional system-based evaluation were keen for a 
dialogue with interactive practitioners and for user models and tasks that can be used within test collection or system-based evaluations. A concern was raised that the core metric of relevance is being stretched too far and we need additional measures to discuss interactive searching. The work of Toms and O’Brien was signaled as a way of defining new metrics from careful user studies. In addition, we should not isolate online searching to search engines alone, but also include other sources of information with different models, such as those used by Amazon.com. There was also recognition that the process of searching is as important as the end-product of searching.

Throughout the workshop the discussion demonstrated that Web Information Seeking and Interaction is both a complex area and one that requires input from many sources. We are, therefore, grateful for the input from our panelists, attendees, and contributors to the proceedings who gave us a lively day of discussion and insights from different parts of the community. 